\title{
Durable Goods Rollover Strategy for Product Depreciation
}

\author{
WangNing $^{1, \mathrm{a}}$ Ye Taofeng ${ }^{1, \mathrm{~b}^{*}}$ \\ ${ }^{1}$ Department of management science and Engineering, Jiangsu University of Science and Technology, Zhenjiang, Jiangsu, \\ China \\ ${ }^{a}$ wangning_seven77@foxmail.com \\ $b^{*} y t f \_j j @ 163 . c o m$
}

\begin{abstract}
This article studies the impact of product depreciation on the product rollover and pricing strategies of durable goods manufacturers under different degrees of product innovation. Based on strategic consumers, the article divides product innovation into two cases: general innovation and major innovation. It analyzes the balanced decision of the firm under different levels of innovation and different rollover strategies, and then draws the optimal rollover strategy for each level of innovation. The research finds that: in general innovation, the equilibrium decision of the firm is affected by the level of product innovation and product depreciation. At the same time, when choosing single product rollover strategy, we must carefully consider the impact of product innovation levels. For major innovations, the single rollover strategy is always better than the dual rollover strategy. In addition, the dual rollover is not affected by the level of product innovation.
\end{abstract}

Keywords: single rollover, dual rollover, product depreciation, strategic consumer

\section{考虑产品折旧的耐用品换代策略}

\author{
王宁 $1, \mathrm{a}$ 叶涛锋 $1, \mathrm{~b}^{*}$
}

${ }^{1}$ 江苏科技大学管理科学与工程系, 镇江, 江苏, 中国

${ }^{a}$ wangning_seven77@foxmail.com

$b^{*} y t f j j @ 163 . c o m$

\section{摘要}

本文研究不同产品创新程度下，考虑产品折旧对耐用品制造商的产品换代与定价策略的影响。文中基 于策略型消费者, 将产品创新分为一般创新和重大创新两种情况, 分析企业在不同创新程度、不同换 代策略下的均衡决策，据此得出每种创新程度下的最优换代策略。研究发现：一般创新时，企业均衡 决策受产品创新水平和产品折旧的影响。同时, 选择单品换代策略时要仔细考虑产品创新水平的影响。 重大创新时, 单品换代策略总是优于共生换代策略。此外, 共生换代策略不受产品创新水平的影响。 关键词：单品换代；共生换代；产品折旧；策略型消费者

\section{1. 前言}

激烈的市场竞争和复杂的环境使得企业在进行产 品定价和换代策略选择时面临更多的问题。在此背景下, 本文研究了产品折旧如何影响企业在不同创新水平下 的耐用品定价和最优换代策略的选择。这与 Dou 等人 ${ }^{[1]}$ 的研究相似, 考虑了消费者策略性程度, 产品折旧与对 产品定价和耐用品换代策略的影响。但与他们的研究不 同的是, 本文除了一般创新, 还考虑了产品创新为重大
创新的情况。对于产品的更新换代, Levinthal 和 Purohit [2]研究了单品换代, 共生换代和旧产品回购三种策略下 新旧产品的需求和竞争蚕食问题; Purohit ${ }^{[3]}$ 在他们的基 础上增加了产品内生创新与换代竞争; ; Liang 等人 ${ }^{[4]}$ 研 究了产品换代策略与消费者行为的相互作用。消费者的 策略性行为也更具现实意义和理论意义, 例如 $\mathrm{Li}$ 等人 ${ }^{[5]}$, Soyal 和 Krishnamurthi ${ }^{[6]}$ 的文献中研究了消费者的策略 性行为对产品定价与换代决策的作用。在我们的研究中, 消费者是策略型消费者, 他们在第一个销售季节开始时 到达市场，然后根据对产品的估值和未来价格的预期以 
$\mathrm{LN} 、 \mathrm{NH}$ 和 $\mathrm{NN}$ 。根据第二章的效用函数, 通过设定 $u_{L H}=u_{\mu}$ 得到 $\theta_{b}=\frac{P_{H}}{\beta-\phi}$ 。设定 $u_{L N}=u_{N H}$ 得到 $\theta_{W H}=\frac{P_{L}-\delta P_{H}}{1+\delta \phi-\delta \beta}$, 设定 $u_{N H}=u_{N N}$ 得 到 $\theta_{i n}=\frac{P_{\mu}}{\beta}$ 。因此, 当均衡状态下 NN, NH, LN 和 LH 同时存 在时, 可能的消费者细分结构是 $\{N N, N H, L N, L H\}$ 。但 是, 当产品 $\mathrm{L}$ 和 $\mathrm{H}$ 的价格偏高或偏低时, $\mathrm{LN}$ 和 $\mathrm{NH}$ 不能 共存。

引理 1. 在一般创新和单品换代策略下, 消费者细 分结构是

$$
\left\{\begin{array}{cc}
\{N N, L N, L H\} & , \quad P_{L} \leq \frac{(1+\delta \phi) P_{H}}{\beta} \\
\{N N, N H, L N, L H\} & , \quad \frac{(1+\delta \phi) P_{H}}{\beta}<P_{L} \leq \frac{P_{H}}{\beta-\phi} \\
\{N N, N H, L H\} & , \\
P_{L}>\frac{P_{H}}{\beta-\phi}
\end{array}\right.
$$

引理 1 表明, 消费者细分结构完全取决于第一期的 产品 $\mathrm{L}$ 的价格水平。在均衡状态下, 只有当第一期的产 品 $\mathrm{L}$ 的价格中等时, 只在第一期购买或只在第二期购买 的消费者才会共存。这是因为 $P_{L}$ 处于中等时, 表明消费 者对当期产品的估值不低, 未来价格的预期也不高。因 此, 当消费者对当期产品的估值较高而对未来价格预期 较低时, 只在第一期购买的消费者会选择在第二期购买, 以获得更高的产品价值; 反之, 当产品估值较低, 但未 来价格预期较高时, 显然只在第一期购买比只在第二期 购买更有利可图。由于消费者的异质性和消费者之间的 相互作用是被忽略不计的, 所以在所有消费者细分结构 下, 都有消费者选择既在第一期购买产品 $\mathrm{L}$, 又在第二 期购买创新产品 $\mathrm{H}$; 还有的消费者综合考虑后选择在两 个销售季节结束后空手离开市场。

基于引理 1 , 我们可以通过三种可能的消费者细分 结构的均衡总利润的比较, 得到单品换代下的均衡决策, 结论如下:

引理 2. (1) $0<\phi \leq \frac{1}{2}$ 时, 企业最优均衡价格分 别是 $P_{H}^{R}=\frac{\beta-\phi}{2}$ 和 $P_{L}^{R}=\frac{(1+\delta \phi)(\beta-\phi)}{2 \beta}$, 相应的企业均衡总利 润是 $\pi^{R}=\frac{1+\delta \beta}{4}-\frac{\phi^{2}(1+\delta \phi)}{4 \beta^{2}}$ 。

( 2 ) $\frac{1}{2}<\phi<1$ 时, 分别 定义 $\delta_{1}=\frac{(2 \phi-\beta)(2 \beta-\phi)}{(\beta-\phi)\left(4 \beta \phi-\beta^{2}-2 \phi\right)}$, $\delta_{2}=\frac{4 \phi^{2}(2 \beta-\phi)-\beta^{2}(\beta-3 \phi)}{\beta^{2}\left(\beta^{2}+\beta \phi+\phi^{2}\right)-4 \phi^{3}(2 \beta-\phi)}, \quad \beta_{1} \in(1,2 \phi] \quad, \quad \beta_{2} \in\left(2 \phi, \frac{1+\delta \phi}{\delta}\right]$. $4 \phi^{3}(2 \beta-\phi \varphi)-\beta^{2}\left(\beta^{2}+\beta \phi+\phi^{2}\right)=0$ 和 $4 \phi^{2}(2 \beta-\phi)-\beta^{2}(\beta-3 \phi)=0$ 有根。企业的最优均衡价格 
覆盖。

$\left(P_{H}^{R}, P_{L}^{R}\right)$ 是

$\left(\frac{\beta(\beta-\phi)[\delta \beta(\beta-\phi)+3(2 \beta-\phi)(1+\delta \phi-\delta \beta)]}{(2 \beta-\phi)[\delta \beta(\beta-\phi)+2(2 \beta-\phi)(1+\delta \phi-\delta \beta)]}, \frac{\delta \beta(\beta-\phi)}{2 \beta-\phi}+\frac{2(2 \beta-\phi)(1+\delta \phi-\delta \beta)^{2}}{\delta \beta(\beta-\phi)+4(2 \beta-\phi)(1+\delta \phi-\delta \beta)}\right)$

, $\beta<\beta \leq 2 \phi, \delta \leq \delta_{1}$;

$\left(\frac{\beta(\beta-\phi)}{3 \beta-2 \phi}, \frac{\beta}{3 \beta-2 \phi}\right),(a) \beta \leq \beta_{1}(b) \beta_{1}<\beta \leq 2 \phi, \delta_{1}<\delta \leq \delta_{2}(c) 2 \phi<\beta \leq \beta_{2}, \delta \leq \delta_{2} ;$

$\left(\frac{\beta-\phi}{2}, \frac{(1+\delta \phi)(\beta-\phi)}{2 \beta}\right), \quad(a) \beta<\beta \leq \beta_{2}, \delta>\delta_{2}(b) \beta>\beta_{2}$ 。

相应地, 企业两期最优均衡总利润 $\pi^{R}$. 为

$\frac{\delta \beta(\beta-\phi)}{2 \beta-\phi}+\frac{(2 \beta-\phi)(1+\delta \phi-\delta \beta)^{2}}{\delta \beta(\beta-\phi)+4(2 \beta-\phi)(1+\delta \phi-\delta \beta)}$,

$\beta_{1}<\beta \leq 2 \phi, \delta \leq \delta_{1}$;

$\frac{\beta(\beta-\phi)[2+\delta(2 \beta-\phi)]}{(3 \beta-2 \phi)^{2}}, \quad(a) \beta \leq \beta_{1}(b) \beta_{1}<\beta \leq 2 \phi, \delta<\delta \leq \delta \delta_{2}(c) 2 \phi<\beta \leq \beta_{2}, \delta \leq \delta$;

$\frac{1+\delta \beta}{4}-\frac{\phi^{2}(1+\delta \phi)}{4 \beta^{2}}, \quad(a) \beta<\beta \leq \beta_{2}, \delta>\delta_{2}(b) \beta>\beta_{2}$ 。

从引理 2 可以看出, 产品折旧影响企业均衡决策。 当产品折旧较小, 即 $0<\phi \leq \frac{1}{2}$ 时, 企业均衡决策具有唯 一性, 即 $\{N N, N H, L H\}$ 。这是因为当产品折旧较低时, 表明产品过时产生的剩余价值较低, 因此选择只在第一 期购买 $L$ 的消费者会选择等待, 在第二期购买产品 $H$ 。

当产品折旧较大, 即 $\frac{1}{2}<\phi<1$ 时, 企业均衡决策由利润 折扣 $\delta$ 与产品创新水平 $\beta$ 决定。

\section{2, 共生换代策略}

共生换代策略下可能的消费者购买行为和消费者 效用有以下五种情形 LH、LN、NH、NL 和 NN。与单品换 代的分析相似, 我们得到均衡状态下, 可能的消费者细 分结构是

$$
\left\{\begin{array}{ccc}
\{N N, N L, L N, L H\}, & P_{L 2} \leq \frac{\phi P_{H}}{\beta}, & P_{L 1} \leq \frac{P_{H}-(1+\delta \phi-\delta \beta) P_{L 2}}{\beta-\phi} \\
\{N N, N L, N H, L N, L H\}, & P_{L 2} \leq \frac{\phi P_{H}}{\beta}, & \frac{P_{H}-(1+\delta \phi-\delta \beta) P_{L 2}}{\beta-\phi}<P_{L 1} \leq \frac{P_{H}}{\beta-\phi} \\
\{N N, N L, N H, L H\}, & P_{L 2} \leq \frac{\phi P_{H}}{\beta}, & P_{L 1}>\frac{P_{H}}{\beta-\phi}
\end{array}\right.
$$

显然, 只有当第二期销售产品 $\mathrm{L}$ 的价格足够低, 共 生换代策略才能存在。同时, 只有产品 $\mathrm{L}$ 的第一期销售 价格处于中等时, NH 和 LN 部分才能共存。当产品 L 的 第一期销售价格较高或较低时, 都可能导致 $\mathrm{NH}$ 部分的 消费者数量向 LN 倾斜或 LN 部分的消费者数量向 NH 倾 斜, 最后 NH 部分被 LN 部分覆盖, 或 LN 部分被 $\mathrm{NH}$ 部分
根据上述消费者细分结构, 通过三种不同细分结构 下的均衡利润的比较, 推导出企业在共生换代策略下的 均衡决策。结论如引理 3 所示:

引理 3. 一般创新和共生换代策略下，企业最优均 衡价格是 $P_{H}^{L}=\frac{\beta-\phi}{2}, P_{L i}^{L}=\frac{(2+\delta \phi)^{2}}{2(4+\delta \phi)}$ 和 $P_{L 2}^{L}=\frac{\phi(2+\delta \phi)}{2(4+\delta \phi)}$ 。企业最 优均衡总利润是 $\pi^{L}=\frac{\delta(\beta-\phi)}{4}+\frac{(2+\delta \phi)^{2}}{4(4+\delta \phi)}$ 。

从引理 3 可以看出, 产品 $\mathrm{H}$ 的价格由创新水平 $\beta$ 和 产品折旧 $\phi$ 决定, $\beta$ 越大或 $\phi$ 越小, $P_{H}^{L}$ 的值越大。这是 因为当创新水平越大, 产品 $\mathrm{H}$ 对消费者吸引力更大, 企 业会对 $\mathrm{H}$ 收取一个较高的价格; 当产品折旧越小，即旧 产品 $\mathrm{L}$ 过时产生的剩余价值较低, 消费者更愿意购买产 品 $\mathrm{H}$ 。可能购买产品 $\mathrm{H}$ 的消费者数量增加, 企业将会对 $\mathrm{H}$ 收取较高的价格以获得更多的利润。共生换代策略下 的均衡决策具有唯一性, 即 $\{N N, N L, L N, L H\}$ 。当产品 $\mathrm{L}$ 的第一期销售价格处于中等或较高时, 产品 $\mathrm{L}$ 在第一 期对消费者的吸引力降低, 在第一期购买产品 $\mathrm{L}$ 的消费 者数量减少。为了获得更多的利润, 企业会尽可能降低 产品 $\mathrm{H}$ 的价格, 第一期未购买的消费者会选择在第二期 购买产品 $\mathrm{H}$ 而不是 $\mathrm{L} 。$ 换句话说, 第二期时, 企业不需 要提供产品 $\mathrm{L}$ 。因此, 当 $P_{L 2} \leq \frac{\phi P_{H}}{\beta}$ 和 $P_{L 1}>\frac{P_{H}-(1+\delta \phi-\delta \beta) P_{L 2}}{\beta-\phi}$ 时, 共生换代策略转化为单品换 代策略。

\section{3，最优策略}

基于引理 2 和引理 2 , 我们可以通过两种不同换代 策略下的均衡总利润的比较, 我们得到一般创新时企业 的均衡决策是

命 题 1. 定义 $\beta_{3}=\sqrt{\frac{\phi(1+\delta \phi)(4+\delta \phi)}{\delta}}$ ， $\phi_{1} \in\left(\frac{1}{2}, 1\right)$ 。一般创新下, 根据 $\phi$ 的取值范围和 $\beta_{2}, \beta_{3}$ 的关系我们得到以下结论:

（1） $0<\phi \leq \frac{1}{2}$ 时, 共生换代策略总是优于单品换 代策略;

( 2 ) $\frac{1}{2}<\phi \leq \phi_{1} \circ$ (i) $\beta_{2}>\beta_{3} \circ(a) \beta_{1}<\beta \leq 2 \phi, \delta \leq \delta_{1}$ 或 (b) $\beta_{3}<\beta \leq \beta_{2}, \delta \leq \delta$ 时, 单品换代策略优于共生换代策略; (a) $\beta \leq \beta_{1},(b) \beta_{1}<\beta \leq 2 \phi, \delta_{1}<\delta \leq \delta_{2} \quad, \quad(c) 2 \phi<\beta \leq \beta_{3}, \delta \leq \delta_{2} \quad$ ， $(d) \beta_{1}<\beta \leq \beta_{2}, \delta>\delta_{2},(e) \beta>\beta_{2}$ 时, 共生换代策略优于单品换代 
产品 $\mathrm{H}$ 的价格随产品创新水平的提高而提高, 产品 $\mathrm{H}$ 创 新水平提高也使得企业能够获得更多的利润。然而, 随 之创新水平提高, 产品 $\mathrm{H}$ 的价格逐渐升高, 选择购买 $\mathrm{H}$ 的消费者会逐渐减少，相应地，消费者细分结构 $\{N N, N H, L H\}$ 的利润也逐渐低于 $\{N N, L N, L H\}$ 的利 润。因此, 重大创新和单品换代策略下, 企业的均衡决 策具有唯一性, 即 $\{N N, L N, L H\}$ 。

通过比较引理 2 和引理 5 , 我们发现一般创新时, 产品折旧和创新水平影响企业的均衡决策选择。重大创 新时, 企业均衡定价和换代决策完全由产品创新水平决 定。

\section{2，共生换代策略}

品折旧较大时，根据产品创新水平和第二期利润折扣的 取值范围不同, 所采取的换代策略也不同。第二期的销 售利润折扣特别低时，单品换代策略更有利可图。

\section{4. 重大创新}

与一般创新一样, 先分析单品换代和共生换代策略 的均衡决策, 再通过两种不同换代策略下均衡总利润的 比较, 推导出企业的最优换代策略。

\section{1 , 单品换代策略}

$$
\text { 给定 } \beta>\frac{1+\delta \phi}{\delta} \text {, 我们有 }
$$
$u_{L N}-u_{N H}=(1+\delta \phi-\delta \beta) \theta-P_{L}+\delta P_{H}\left\{\begin{array}{ll}\geq 0, & \theta \leq \theta_{l h} \\ \leq 0, & \theta \geq \theta_{l h}\end{array}\right.$ 其 中 $\theta_{l h}=\frac{\delta P_{H}-P_{L}}{\delta \beta-1-\delta \phi}, \mathrm{LN}$ 在 $\mathrm{NH}$ 的右边。

与引理 1 相似, 我们的重大创新下, 单品换代策略 的消费者细分结构为

引理 4. 在重大创新和单品换代策略下, 可能的消 费者细分结构是

$$
\begin{cases}\{N N, L N, L H\}, & \mathrm{P}_{L} \leq \frac{(1+\delta \phi) P_{H}}{\beta} \\ \{N N, N H, L H\}, & \mathrm{P}_{L}>\frac{(1+\delta \phi) P_{H}}{\beta}\end{cases}
$$

引理 4 分析与第三章中重大创新下的单品换代策略 的消费者细分结构的分析相似。

通过比较引理 4 中不同消费者细分结构下的均衡总 利润, 我们得到以下结论:

引理 5. 重大创新和单品换代策略下, 企业最优均 衡价格是 $P_{H}^{R_{.}}=\frac{\beta}{2}$ 和 $P_{L}^{R}=\frac{1}{2}$, 两期最优均衡总利润是 $\pi^{R .}=\frac{1+\delta \beta}{4} 。$

从引理 5 可以知道, 有半数消费者选择购买产品 $\mathrm{L}$ 。
给定 $\beta>\frac{1+\delta \phi}{\delta}$, 我们有 $u_{L N}-u_{N H}=(1+\delta \phi-\delta \beta) \theta-P_{L 1}+\delta P_{H}\left\{\begin{array}{ll}\geq 0, & \theta \leq \theta_{u h} \\ \leq 0, & \theta \geq \theta_{u h}\end{array}\right.$, 其 中 $\theta_{u h}=\frac{\delta P_{H}-P_{L 1}}{\delta \beta-1-\delta \phi}, \mathrm{LN}$ 在 NH 的右边。

与一般创新下共生换代的消费者细分结构相似, 我 们得到

$\begin{cases}\{N N, N L, L N, L H\}, \quad \mathrm{P}_{L 2} \leq \frac{\phi P_{H}}{\beta}, \frac{(1+\delta \phi) \mathrm{P}_{L 2}}{\phi}<\mathrm{P}_{L 1} \leq \frac{P_{H}+(\delta \beta-1-\delta \phi) \mathrm{P}_{L 2}}{\beta-\phi} \\ \{N N, N L, N H, L H\}, \quad \mathrm{P}_{L 2} \leq \frac{\phi P_{H}}{\beta}, \quad \mathrm{P}_{L 1}>\frac{P_{H}+(\delta \beta-1-\delta \phi) \mathrm{P}_{L 2}}{\beta-\phi}\end{cases}$

与一般创新的情况不同, 消费者购买 $\mathrm{L}$ 或 $\mathrm{H}$ 取决 于哪个产品的价格更低。回顾一般创新的分析, LN 和 $\mathrm{NH}$ 只有在 $\mathrm{L}$ 和 $\mathrm{H}$ 的价格都处于中等时才能并存。当产 品 $\mathrm{H}$ 的创新水平相当高时, 企业也会对 $\mathrm{H}$ 收取更高的 价格。这导致想要在第二期购买 $\mathrm{H}$ 的消费者转而在第一 期购买 $L$ 。

基于上述消费者细分结构, 我们可以推导出重大创 新和共生换代策略下的企业均衡决策是

引理 6. 重大创新下, 共生换代策略的企业均衡决 策与一般创新时一样。

结合引理 3 和引理 6 , 我们发现考虑产品折旧因素 的影响后, 企业的产品创新水平不能影响到企业共生换 代策略的选择。

\section{3，最优策略}

与一般创新分析相似, 通过两种不同的换代策略的 均衡总利润的比较, 我们得到重大创新下企业的最优均 衡换代策略。

命题 2. 重大创新下，单品换代策略始终优于共生 换代策略。

在重大创新下, 产品 $\mathrm{L}$ 在两期的价格都较低, 但随 着产品创新水平的提高, 产品 $\mathrm{H}$ 的价格也随之提高。所 以单品换代策略下 $\mathrm{NH}$ 部分获得的利润大于共生换代策 
[9] Shum S., Tong S.L., Xiao T.T. (2017) On the impact of uncertain cost reduction when selling to strategic customers. J. Management Science, 63: 843-860.

[10] Papanastasiou Y., Savva N. (2017) Dynamic pricing in the presence of social learning and strategic consumers. J. Management Science, 63: 919-939.

文章讨论了企业在产品创新程度为低中创新和重 大创新时, 对单品换代与共生换代两种换代策略的选择 问题。我们发现: 一般创新时, 产品折旧和创新水平会 影响企业均衡决策的选择。在重大创新时, 单品换代始 终优于共生换代。共生换代策略在两种创新水平下的定 价与消费者细分结构都具有唯一性, 且两种情况下的企 业均衡总利润相同。企业在选择单品换代时, 要仔细考 虑产品的创新水平, 共生换代策略则不需要考虑产品创 新。

\section{项目基金}

本文为江苏省科研创新项目《技术进步条件下耐用 品引入与定价策略研究》 (项目号: KYCX19_1653) 的阶 段性成果之一。

\section{REFERENCES}

[1] Dou Y.F., Hu Y.J., Wu D.J. (2017) Selling or Leasing? Pricing Information Goods with Depreciation of Consumer Valuation. J. Social Science Electronic Publishing, 28: 585602.

[2] Levinthal D.A., Purohit D. (1989) Durable goods and product obsolescence. J. Marketing Science, 8: 35-35.

[3] Purohit D. (1994) What should you do when your competitors send in the clones? J. Marketing Science, 13: $392-411$.

[4] Liang C., Cakanyildirim M., Sethi, S.P. (2014) Analysis of product rollover strategies in the presence of strategic customers. J. Management Science, 60: 1033-1056.

[5] Li J., Granados N., Netessine S.(2014) Are consumers strategic? Structural estimation from the air-travel industry. J. Management Science, 60: 2114-2137.

[6] Soysal G.P., Krishnamurthi L. (2012) Demand dynamics in the seasonal goods industry: An empirical analysis. J. Marketing Science, 31: 293-316.

[7] Cachon G.P., Swinney R. (2011) The value of fast fashion: Quick response, enhanced design, and strategic consumer behavior. J. Management Science, 57: 778-795.

[8] Swinney R. (2011) Selling to strategic consumers when product value is uncertain: The value of matching supply and demand. J. Management Science, 57: 1737-1751.
[11] Cachon G.P., Swinney R. (2009) Purchasing, pricing, and quick response in the presence of strategic consumers. J. Management Science, 55: 497-511.

[12] Su X., Zhang F. (2008) Strategic consumer behavior, commitment , and supply chain performance. J. Management Science, 54:1759-1773.

[13] Mantena R., Tilson V., Zheng X. (2012) Literature survey: Mathematical models in the analysis of durable goods with emphasis on information systems and operations management issues. J. Decision Support Systems, 53:331344. 